\title{
Comportamento e padrão alimentar de uma espécie de Latrodectus do grupo Mactans (Araneae, Theridiidae) em cativeiro
}

\author{
Maria de Fátima da Rocha Dias ${ }^{1}$ \\ Tania Kobler Brazil ${ }^{1}$
}

\begin{abstract}
Behavior and diet supply of Latrodectus group Mactans (Araneae, Theridiidae) in captivity. Latrodectus gr. mactans is responsible for $28 \%$ of all accidents provoked by spiders in state of Bahia, Brazil (1980-1990), which makes necessary the study of its manejament. The spiders were captured in Ondina, Salvador, and in Baxio, Esplanada (Bahia, Brazil); they were mantained in captivity, with food supply weekly (Atta sp., larva of Tenebrio sp. and Drosophila melanogaster) with three hours/ offers observation time, during march/95 to april/96. In captivity, the spiders accepted two kinds of food: Atta sp., which seems to be the major item of its diet in natural conditions, and larva of Tenebrio sp., which is not available in natural conditions. Drosophila melanogaster was systematically rejected. The feeding behavior is composed by four distinct steps: (1) immobilization, (2) inoculation, (3) second immobilization and (4) ingestion. Two kinds of social alimentary behavior are described. The results indicated that larvae of Tenebrio sp. can be an alternative supply for successfull maintenance of this specie in captivity.

KEY WORDS. Araneae, Theridiidae, Latrodectus, feed, diet, behavior
\end{abstract}

Latrodectus grupo mactans (ABALOS \& BÁEZ 1967) é uma aranha de interesse médico, responsável por acidentes em seres humanos no estado da Bahia (Brasil); no período de 1980 a 1990, foram registrados 77 casos de latrodectismo no Estado, o equivalente a $28 \%$ dos acidentes araneídicos (LIRA-DA-SILVA et al. 1995). O soro anti-latrodectico (SALAT) ainda não é produzido no Brasil, sendo importado da Argentina para atender os casos de latrodectismo no país (MINISTÉRIO DA SAÚDE 1992).

LIRA-DA-SILVA et al. (1995) atribuíram os casos de latrodectismo na Bahia a Latrodectus curacaviensis Müller, 1776 (identificada erroneamente), porém, o estudo da morfologia da genitália de machos e fêmeas, indicou que a espécie pertence ao grupo mactans, caracterizado por apresentar o epígino com ductos e o êmbolo do palpo do macho com três espiras (ABALOS \& BÁEZ 1967; ABALOS 1980). A identificação específica depende ainda de um estudo revisivo que abranja todas as espécies do grupo mactans na região Neotropical. Aspectos da biologia das Latrodectus spp. ainda são pouco estudados, principalmente quanto ao comportamento alimentar (KASTON 1970). Os poucos trabalhos que existem referem-se a

1) Laboratório de Animais Peçonhetos, Departamento de Zoloogia, Instituto de Biologia, Universidade Federal da Bahia. Campus Universitário de Ondina, 40170-210 Salvador, Bahia, Brasil.

Email: lap-zoo@ufba.br 
Latrodectus mactans (Argentina, Estados Unidos da América), abordando apenas a dieta dessas aranhas (KASTON 1970; GonZALEZ 1977, 1979; SCHENONE \& CORREA 1985; FORSTER 1995).

Considerando a importância médica desta aranha e as poucas informações sobre a sua biologia, este trabalho tem o objetivo de fornecer informações básicas sobre o seu comportamento e padrão alimentar para que subsidiem o seu manejo em cativeiro, principalmente para fins de extração de veneno.

\section{MATERIAL E MÉTODOS}

As aranhas e as ootecas foram capturadas no campus da Universidade Federal da Bahia (UFBA) no bairro de Ondina, Salvador e em Baixio, Esplanada ambos na Bahia, Brasil, onde foram realizadas mensalmente as observações de campo (média de três horas por observação). Foram mantidas em cativeiro no Laboratório de Animais Peçonhentos (LAP) do Departamento de Zoologia do Instituto de Biologia (UFBA), onde realizaram-se as observações e experimentos com as ninhadas ovipostas e/ou eclodidas em laboratório. Foram analisadas 15 ootecas, 11 ninhadas, sendo quatro mantidas com fêmeas e sete sem fêmeas, totalizando 373 indivíduos; quatro ootecas eclodiram juntas, sendo consideradas uma única ninhada. Os machos foram separados logo após a maturação sexual, para evitar intercruzamentos.

Para os terrários foram utilizados frascos tipo maionese, cobertos com pano descartável tipo Perfex ${ }^{\circledR}$ e seguro com elástico. A umidade foi mantida através do borrifamento semanal com água.

Foram oferecidos três tipos de alimento: formiga saúva cortadeira, Atta sp. (Hymenoptera, Formicidae), larvas de Tenebrio sp. (Coleoptera, Tenebrionidae) e drosófilas, Drosophila melanogaster (Diptera, Drosophilidae), de maneira alternada, uma vez por semana, pela manhã, no período de março de 1995 a abril de 1996, com tempo de observação de três horas para cada oferta.

Para facilitar a análise do comportamento alimentar foram estipulados sete passos que indicassem cada tipo de comportamento observado: A) imobilização, inoculação, segunda inoculação, ingestão; B) imobilização, inoculação, segunda inoculação, sem ingestão; C) "social" (matam e comem juntos); D) "social" (matam juntos e comem separados); E) "Maternidade" (fêmea com iniciativa); F) "maternidade" (filhotes com iniciativa); G) canibalismo.

O material testemunho está depositado em coleções científicas do Departamento de Zoologia do Instituto de Biologia, UFBA (curadora: Tania Kobler Brazil) e do Instituto Butantan (curador: Antonio D. Brescovit).

\section{RESULTADOS}

As observações mostraram que adultos da espécie em estudo alimentaram-se tanto na natureza, quanto em cativeiro de Atta sp. (formiga saúva). As formigas foram encontradas presas nas teias, que estavam sempre em local próximo a formigueiros. Em cativeiro, adultos e jovens alimentaram-se também de larvas de Tenebrio sp.. Drosofila melanogaster foi testada em três lotes durante três semanas, não sendo aceita por nenhum deles. 
Fêmeas com filhotes e/ou filhotes sem fêmeas, levaram cerca de 24 horas para se alimentar totalmente (ingestão completa) de uma larva de Tenebrio sp. e menos de quatro horas para alimentar-se de uma Atta sp.. Em nenhum momento foi observado a retirada de restos de alimento da teia.

Durante o processo de apreensão de alimento as fêmeas foram mais rápidas do que os machos, sendo observados os seguintes comportamentos:

\section{A) Imobilização, inoculação, segunda imobilização, ingestão (filhotes e adultos)}

Quando o alimento era colocado no terrário com a pinça e tocava a teia, as aranhas percebiam sua vibração e se dirigiam ao local, tocando-o com seu primeiro par de pernas; davam uma volta e ficavam de costas para a presa e com o último par de pernas, começavam a tecer com rápidos movimentos alternados das pernas até envolvê-la com a seda. Após a primeira imobilização, era feita a inoculação de veneno sempre nas articulações (no caso de Atta sp.) e em seguida, as aranhas começavam a se alimentar pela cabeça; se fosse uma larva de Tenebrio sp., porém, a inoculação dava-se em qualquer parte do corpo, podendo haver várias inoculações do veneno até que a presa cessasse seus movimentos. Após a segunda imobilização, em ambos os casos, a presa era envolvida novamente em seda e só então iniciado o processo de ingestão.

B) Imobilização, inoculação, segunda inoculação, sem ingestão (adultos)

Todo o processo anterior, mas sem se alimentarem.

\section{C) "Social" matam e comem juntos (filhotes até três meses)}

Ao colocar o alimento em número igual ou maior do que o de aranhas, estas se dirigiam ao local onde estavam as presas e em conjunto, começavam a envolvê-las uma a uma com teia, matando-as e alimentando-se, todas ao mesmo tempo.

\section{D) "Social" matam junto e comem separados (filhotes acima de quatro meses)}

O mesmo processo acima, mas sem repartir o alimento, afastando-se umas das outras com a sua presa.

\section{E) "Maternidade" - fêmea com iniciativa (filhotes até sete dias)}

A fêmea imobilizava e inoculava o veneno na presa (Tenebrio sp.) da maneira descrita em A. Após a segunda imobilização, com uma das pernas I dava golpes na teia e os filhotes vinham se alimentar. Com a movimentação da larva de Tenebrio sp. a teia sempre era danificada; enquanto os filhotes estavam se alimentando a fêmea cortava a teia com movimentos circulares, deixando o alimento preso apenas por alguns fios na porção superior do frasco. Fazia duas amarras (uma ligando a teia antiga ao frasco, e outra ligando a presa à teia antiga), voltava a larva de Tenebrio sp. e se alimentava por cerca de 10 segundos com os filhotes, depois deixava-os se alimentando e começava a tecer uma nova teia. Percebia-se claramente que os filhotes estavam se alimentando, pois seus abdomens dobravam de tamanho. A fêmea sempre voltava para o alimento durante a reconstrução da teia, após o que todos se alimentavam juntos. 


\section{F) "Maternidade" - filhotes com iniciativa (filhotes após sete dias)}

Depois da primeira muda, os filhotes tomavam a iniciativa para apreensão do alimento e a fêmea aguardava alguns segundos até alimentar-se.

\section{G) Canibalismo}

Ocorreu em quatro lotes, toda vez que um dos indivíduos não completava a muda, ficando preso pela perna.

O comportamento A foi observado em todos os lotes das ninhadas e o comportamento B em nenhum deles, apenas com os adultos; o comportamento C foi observado em $100 \%$ nas ninhadas jovens até oito semanas, sendo que $55,5 \%$ em ninhadas mais velhas ( 21 à 29 semanas); nenhuma ninhada nova (até 8 semanas) apresentou o comportamento $\mathrm{D}$; todas as ninhadas mantidas com a fêmea apresentaram o comportamento $\mathrm{E}$ e F, a depender da idade dos filhotes; o comportamento $\mathrm{G}$ foi observado em 36,4 \% das ninhadas (10,3-37,5 \% das observações) (Tab. I).

Tabela I. Comportamento alimentar observado nas ninhadas de Latrodectus grupo Mactans.

\begin{tabular}{|c|c|c|c|c|c|c|c|c|c|c|}
\hline \multirow[t]{2}{*}{ Lotes } & \multirow{2}{*}{$\begin{array}{c}\text { Data de } \\
\text { nascimento }\end{array}$} & \multirow{2}{*}{$\begin{array}{l}\text { Número de } \\
\text { individuos }\end{array}$} & \multirow{2}{*}{$\begin{array}{l}\text { Idade das } \\
\text { ninhadas }\end{array}$} & \multirow{2}{*}{$\begin{array}{l}\text { Número de } \\
\text { observaçð̄es }\end{array}$} & \multicolumn{6}{|c|}{ Comportamento alimentar (\%) } \\
\hline & & & & & A & C & $D$ & $E$ & $\mathrm{~F}$ & $G$ \\
\hline 3 & $17 / I V / 1995$ & 40 & 227 & 28 & 100 & 39,3 & 60,7 & 7,1 & 7,9 & 0,0 \\
\hline 4 & $17 / I V / 1995$ & 36 & 216 & 29 & 100 & 48,3 & 41,4 & 6,9 & 37,9 & 10,3 \\
\hline 5 & $17 N / 1995$ & $18^{*}$ & 210 & 24 & 100 & 50,0 & 41,7 & 0,0 & 0,0 & 8,3 \\
\hline 6 & $02 N / 1995$ & $26^{*}$ & 191 & 27 & 100 & 62,9 & 37,0 & 0,0 & 0,0 & 0,0 \\
\hline 7 & $05 / 11 / 1995$ & $11^{\star}$ & 240 & 21 & 100 & 66,7 & 33,3 & 0,0 & 0,0 & 0,0 \\
\hline 9 & 19/11/1996 & $37^{\star}$ & 60 & 7 & 100 & 100,0 & 0,0 & 0,0 & 0,0 & 14,3 \\
\hline 10 & $27 / 1 / 1996$ & 45 & 100 & 8 & 100 & 100,0 & 0,0 & 25,0 & 37,5 & 37,5 \\
\hline 11 & 24/II/1996 & 35 & 60 & 8 & 100 & 100,0 & 0,0 & 75,0 & 25,0 & 0,0 \\
\hline 13 & $10 / 11 / 1996$ & $25^{*}$ & 60 & 8 & 100 & 100,0 & 0,0 & 0,0 & 0,0 & 0,0 \\
\hline $14^{* *}$ & $10 / 11 / 1996$ & $62^{*}$ & 60 & 6 & 100 & 100,0 & 0,0 & 0,0 & 0,0 & 0,0 \\
\hline 17 & $20 / 11 / 1996$ & $38^{*}$ & 60 & 4 & 100 & 100,0 & 0,0 & 0,0 & 0,0 & 0,0 \\
\hline Total & & 373 & & & & & & & & \\
\hline
\end{tabular}

*) Lotes sem mãe; *^) lote com quatro ootecas eclodidas.

\section{DISCUSSÃO}

De uma maneira geral, espécies de Latrodectus (L. mactans, L. pallidus, $L$. tredecimguttatus, L. curacaviensis, L. bishopi $\mathrm{e}$ L. hasselti, L. hesperus) alimentamse de insetos (BÜCHERL 1968; KASTON 1970; SCHENONE \& CORREA 1985; FORSTER 1995), preferindo formigas (GonZALEZ 1977, 1979; SHULOV 1967; ABALOS 1980; MACKAY 1982) e construindo sua teia próxima a formigueiros como observado em L. mirabilis, L. quartus e L. corallinus (ABALOS 1980), comportamento semelhante ao observado com a espécie estudada neste trabalho. Ocasionalmente tem sido registrado captura e alimentação de pequenos vertebrados como lagartos, cobras e camundongos por L. hasselti (ROBERTS 1941; MCKEOWN 1943; FORSTER 1995), necrofagia em $L$. hesperus (Ross 1979) e canibalismo em L. mactans e L. hasselti (SCHENONE \& CORREA 1985; FOSTER 1995).

Algumas experiências de manutenção de Latrodectus em cativeiro ( $L$. hesperus, $L$. variolus, $L$. mactans, $L$. antheratus, $L$. corallinus, $L$. diaguita, $L$. geometricus, L. mirabilis, L. quartus, L. variegatus e L. hasselti) mostram que elas podem ser alimentadas com Drosophila melanogaster (KASTON 1970; GoNZALEZ 1977, 
1979; ABAlOS 1980; ForSTER 1995), embora os espécimes de Latrodectus gr. mactans não tenham aceitado este tipo de alimento. KASTON (1970), já havia testado o uso de Tenebrio sp., com êxito, na alimentação de várias Latrodectus (L. hesperus, L. variolus, L. mactans). A aceitação desta dieta pelos filhotes e adultos de Latrodectus gr. mactans, indica que a larva de Tenebrio sp., pode ser usada como uma alimentação alternativa, já que seu manejo é mais fácil do que o de formigas.

Para se alimentar, as aranhas apresentaram um comportamento específico (A), que parece ser padronizado pelo menos para Latrodectus mactans e Latrodectus gr. curacaviensis, envolvendo a presa com fios de seda e quando esta é imobilizada, inoculando o veneno, sugando-lhe as partes moles (BÜCHERL 1968). As observações deste trabalho complementam as de BÜCHERL $(1968,1972)$, especificando as etapas que se seguem à apreensão do alimento: 1) imobilização, 2) inoculação, 3) segunda imobilização, 4) ingestão. Sugerem ainda que a espécie estudada apresenta um certo grau de sociabilidade, ao repartir o alimento na própria teia com os filhotes, sendo esta uma das condições definidas por KULLMAN (1972) para a existência de sociabilidade nas aranhas. Ao contrário de L. mactans e de L. geometricus (observação pessoal), não retira os restos de alimentos de sua teia, facilitando o reconhecimento do seu item alimentar na natureza.

O comportamento social (C) foi apresentado em $100 \%$ das observações pelos filhotes abaixo de oito semanas independente da presença da fêmea (Tab. I), sugerindo uma compensação em relação ao seu tamanho e fragilidade, o que facilita a apreensão do alimento. Esse comportamento é apresentado até o terceito mês; a partir do quarto mês, as ninhadas passam a apresentar o comportamento social (D). Dessa maneira, quanto mais velha for a ninhada, mais independente ficam os indivíduos, passando a se alimentarem sozinhos e podendo então serem separados em terrários individuais.

Apesar de ABALos (1980) referir-se a L. corallinus como a única espécie que apresenta hábitos maternais (fêmea capturando e preparando a presa para os filhotes se alimentarem), Latrodectus gr. mactans também apresentou o mesmo hábito (comportamento E).

De maneira geral o canibalismo é considerado como comportamento típico do cativeiro, principalmente em aranhas errantes. No entanto, as fêmeas de Latrodectus (sedentárias) praticam o canibalismo quando colocadas em um local comum ou quando os exemplares são colocados em uma mesma teia (SCHENONE \& CORREA 1985), e em filhotes com mais de dez dias (FOSTER 1995). Latrodectus gr. mactans só apresentou este comportamento quando algum indivíduo ficava preso na muda. Alguns estudos mostram que o comportamento de canibalismo é uma estratégia de crescimento para a fêmea de Latrodectus, permitindo a ela um suprimento extra para o seu desenvolvimento. Fêmeas que adotam este comportamento crescem mais rápido e chegam à maturidade mais cedo do que as outras (FOSTER 1995).

AGRADECIMENTOS. Os autores agradecem ao CNPq através do apoio (Bolsa de Iniciação Científica para o primeiro autor). Aos pesquisadores: César Ades, Antonio D. Brescovit, Alexandre B. Bonaldo, Erica H. Buckup, Hilton Japyassu, Carlos Bertim e Walter Cerqueira pela leitura cuidadosa do manuscrito e críticas ao trabalho. 


\section{REFERÊNCIAS BIBLIOGRÁFICAS}

ABALOS, J.W. 1980. Las arañas del genero Latrodectus en la Argentina. Obra del Centenario del Museo de La Prata 6: 29-51.

ABAlos, J.W.; E.C. BÁEZ. 1967. The spider genus Latrodectus in Santiago del Estero, Argentina, p.59-74. In: Animal Toxins - I International Symposium on Animal toxins. Atlantic City, Pergamon Press, 281p.

BÜCHERL, W. 1968. Latrodectus e Latrodectismo na América do Sul. Bioecologia de Latrodectus do grupo curacaviensis nas praias dos Estados do Rio de Janeiro e Guanabara. Rev. Bras. Pesq. Med. 1 (2): 83-88. $1^{\mathrm{a}}$ ed., $158 \mathrm{p}$.

FORSTER, L. 1995. The behavioural ecological of Latrodectus hasselti (Thorell), the Australian Redback Spider (Araneae: Theridiidae): a review. Rec. Western Australian Mus. 52: 13-24.

GonZALEZ, A. 1977. Observaciones bioecológicas sobre una espécie del genero Latrodectus (Walckenaer, 1805) del grupo Mactans de Sierra de la Ventana (Provincia de Buenos Aires, Argentina). (Araneae, Theridiidae). I. Habitat y ciclo de vida. Physis 36 (92): 277-282.

- 1979. Observaciones bioecológicas sobre una espécie del genero Latrodectus (Walckenaer, 1805) del grupo Mactans de Sierra de la Ventana (Provincia de Buenos Aires, Argentina). (Araneae, Theridiidae) III. Desarrollo Post-embrionario. Acta Zoologica Lilloana. 35: 97-103.

Kaston, B.J. 1970. Comparative biology of American Black Widow Spiders.

Trans. San Diego Soc. Nat. Hist. 16 (3): 33-82.

KULLMAN, E. 1972. Evolution of social behaviour in spiders (Araneae; Eresidae and Theridiidae). Amer. Zool. 12: 419.

LIRA-DA-Silva, R.M.L.; G.B. MATOS; R.O. SAMPAiO; T.B. NunES. 1995. Estudo retrospectivo de latrodectismo na Bahia. Rev. Soc. Bras. Med. Trop. 28 (3): 205-210.

MACKAY, W.P. 1982. The effect of predation of western widow spiders (Araneae: Theridiidae) on harvester ants (Hymenoptera: Formicidae). Oecologia 53: 406-411.

McKeown, K.C. 1943. Vertebrate captured by Australian spiders. Proc. Roy. Zool. Soc. New South Wales 11: 17-29.

MINISTÉRIO DA SAÚDE. 1992. Manual de diagnóstico e tratamento de acidentes por animais peçonhentos. Brasília, Fundação Nacional de Saúde, 58p.

RobertS, N.L. 1941. Some notes on Australian spiders. Proc. Roy. Zool. Soc. New Shouth Wales 9: 36-41.

Ross, K.G. 1979. Report of necrophagy in the black widow spiders, Latrodectus hesperus (Araneae: Theridiidae). Jour. Arachnology 9: 109.

SCHENONE, H. \& L.E. CORREA. 1985. Algunos conocimientos prácticos sobre la biología de la araña Latrodectus mactans y el latrodectismo en Chile. Bol. Chil. Parasitol. 48: 18-23.

SHuLOV, A. 1967. Biology and ecology of venomous animals in Israel. Mem. Inst. Butantan. 33: 93-99.

Recebido em 27.II.1998; aceito em 14.IX.1999. 